\title{
THE COHOMOLOGICAL DIMENSION OF STONE SPACES
}

\author{
BY ROGER WIEGAND
}

Communicated by R. S. Pierce, April 10, 1968

The purpose of this note is to announce a few inequalities involving the cohomological (sheaf-theoretic) dimension of locally compact, totally disconnected Hausdorff spaces, herein called Stone spaces. Throughout, $R$ will denote a commutative regular ring with maximal ideal space $X$. (Then $X$ is compact and totally disconnected.) For each ideal $J$ in $R$ let $U[J]$ denote the corresponding open subset of $X$, and for each $R$-module $A$, let $Q(A)$ denote the corresponding sheaf of modules, as defined in [2].

TheOREM 1. $\operatorname{Ext}_{R}^{n}(J, A)$ and $H^{n}(U[J] ; \mathfrak{Q}(A))$ are naturally isomorphic.

THEOREM 2. Let $\mathfrak{F}$ be a sheaf over the Stone space $X$, and let $\mathcal{u}$ be a covering of $X$ consisting of compact open sets. Then the natural maps $H^{n}(\mathcal{U} ; \mathcal{F}) \rightarrow \breve{H}^{n}(X ; \mathcal{F}) \rightarrow H^{n}(X ; \mathcal{F})$ are all isomorphisms.

Let $\operatorname{dim} X$ denote the cohomological dimension of $X$, and $\operatorname{cov} \operatorname{dim} X$ the covering dimension of $X$, based on arbitrary (not necessarily finite) open coverings. (It is not hard to show that for Stone spaces, cov $\operatorname{dim} X \leqq n$ iff $X$ has a compact open cover of order $n$.) Finally, let $h \cdot \operatorname{dim}_{R} J$ denote the homological (projective) dimension of the ideal $J$.

Corollary. $h \cdot \operatorname{dim}_{R} J \leqq \operatorname{dim} U[J] \leqq \operatorname{cov} \operatorname{dim} U[J]$.

Since the only projective $R$-modules are direct sums of principal ideals [1], we see that $h \cdot \operatorname{dim}_{R} J=0$ iff cov $\operatorname{dim} U[J]=0$, and, by the corollary, iff $\operatorname{dim} U[J]=0$. In order to see that equality need not always hold in the corollary, let us define the rank $\rho$ of a space $X$ by agreeing that $\rho(X) \leqq n$ iff $X$ can be written as a union of $\aleph_{n}$ (or fewer) compact sets.

Theorem 3. For any Stone space $X, \operatorname{dim} X \leqq \rho(X)$.

EXAMPLE 1. Let $\Omega$ be the set of countable ordinals, with the order topology. Then $\operatorname{dim} \Omega=1$, but $\operatorname{cov} \operatorname{dim} \Omega=\infty$. (The second assertion may be verified directly; the first then follows from Theorem 3 and the remarks following the corollary.)

The next example shows that the inequality in Theorem 3 cannot be sharpened. 
EXAMPLE 2. For each $n \geqq 0$, let $X_{n}$ be the product of $\boldsymbol{\aleph}_{n}$ copies of a two point space, with a single point deleted. Then $\operatorname{dim} X_{n}=\rho\left(X_{n}\right)=n$. (Pierce [3] has shown that the corresponding maximal ideal in the free Boolean ring on $\boldsymbol{\aleph}_{n}$ generators has homological dimension $n$. Therefore $\operatorname{dim} X_{n}=n$, by Theorem 3 and the corollary.)

Example 3. Let $A_{0}$ (resp. $A_{1}$ ) be the one-point compactification of a discrete space of cardinality $\boldsymbol{\aleph}_{0}$ (resp. $\boldsymbol{\aleph}_{1}$ ). Let $X=A_{0} \times A_{1}$ $-\{(*, *)\}$. Then $\operatorname{dim} X=$ cov $\operatorname{dim} X=\rho(X)=1$. (In fact, it can be shown that $H^{1}\left(X ; Z_{2}\right) \neq 0$, where $Z_{2}$ denotes the constant 2 -sheaf.)

I do not know whether the identity $\operatorname{dim} X=\operatorname{cov} \operatorname{dim} X=n$ can be realized in general. (The space $X_{n}$ of Example 2 has infinite covering dimension.) An obvious generalization of Example 3 yields a space with rank and covering dimension $n$, but with unknown cohomological dimension. Also, I know of no example in which $h \cdot \operatorname{dim}_{R} J$ $<\operatorname{dim} U[J]$. Notice that if one could show that $h \cdot \operatorname{dim}_{R} J$ and $\operatorname{dim} U[J]$ are always equal, then it would follow that any two commutative regular rings with homeomorphic maximal ideal spaces have the same global dimension.

\section{REFERENCES}

1. I. Kaplansky, Projective modules, Ann. of Math. 68 (1958), 372-377.

2. R. S. Pierce, Modules over commutative regular rings, Mem. Amer. Math. Soc. No. 70 (1967).

3. R. S. Pierce, The global dimension of Boolean rings, J. Algebra 7 (1967), 91-99.

UNIVERSITY OF WISCONSIN 